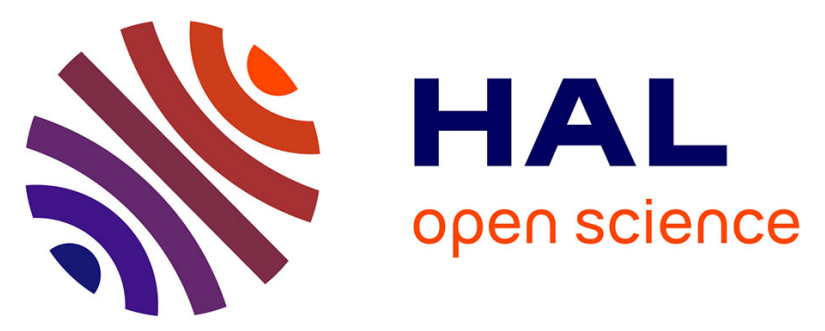

\title{
Type 2 Diabetes Remission After Gastric Bypass: What Is the Best Prediction Tool for Clinicians?
}

Aurélie Cotillard, Christine Poitou, Guillemette Duchâteau-Nguyen, Judith Aron-Wisnewsky, Jean-Luc Bouillot, Thomas Schindler, Karine Clément

\section{To cite this version:}

Aurélie Cotillard, Christine Poitou, Guillemette Duchâteau-Nguyen, Judith Aron-Wisnewsky, JeanLuc Bouillot, et al.. Type 2 Diabetes Remission After Gastric Bypass: What Is the Best Prediction Tool for Clinicians?. Obesity Surgery, 2014, 25 (7), pp.1128-1132. 10.1007/s11695-014-1511-8 . hal01102887

\section{HAL Id: hal-01102887 \\ https://hal.sorbonne-universite.fr/hal-01102887}

Submitted on 13 Jan 2015

HAL is a multi-disciplinary open access archive for the deposit and dissemination of scientific research documents, whether they are published or not. The documents may come from teaching and research institutions in France or abroad, or from public or private research centers.
L'archive ouverte pluridisciplinaire HAL, est destinée au dépôt et à la diffusion de documents scientifiques de niveau recherche, publiés ou non, émanant des établissements d'enseignement et de recherche français ou étrangers, des laboratoires publics ou privés. 


\section{Title page}

\section{Full title}

Type 2 diabetes remission after gastric bypass: what is the best prediction tool for clinicians?

\section{Original contribution}

\section{Authors and affiliations}

Aurélie Cotillard $\mathrm{PhD}^{1,2,3}$, Christine Poitou MD $\mathrm{PhD}^{1,2,3}$, Guillemette Duchâteau-Nguyen $\mathrm{PhD}^{4}$, Judith Aron-Wisnewsky MD $\mathrm{PhD}^{1,2,3}$, Jean-Luc Bouillot MD PhD ${ }^{5}$, Thomas Schindler $\mathrm{PhD}^{4}$, Karine Clément $\mathrm{MD} \mathrm{PhD}^{* 1,2,3}$

A Cotillard (aurelie.nutriomics@gmail.com), C Poitou (christine.poitoubernert@psl.aphp.fr), J Aron-Wisnewsky (judith.aron-wisnewsky@psl.aphp.fr) and K Clément (karine.clement@psl.aphp.fr) Institute of Cardiometabolism and Nutrition, ICAN, Assistance Publique Hôpitaux de Paris, Pitié-Salpêtrière Hospital, 75013, Paris, France

Sorbonne Universités, Université Pierre et Marie Curie-Paris 6, UMR_S U1166, Nutriomics, 75013, Paris, France

INSERM, UMR S U1166, Nutriomics, 75013, Paris, France

G Duchâteau-Nguyen (guillemette.duchateau-nguyen@roche.com) and T Schindler (thomas.schindler@roche.com)

Roche Innovation Center Basel, Pharmaceutical Sciences, 4070, Basel, Switzerland JL Bouillot (j1.bouillot@apr.aphp.fr) 
Assistance Publique-Hôpitaux de Paris, Ambroise Paré Hospital, Surgery Department, 92100, Boulogne-Billancourt, France

\section{Correspondence to}

Karine Clément

Institute of Cardiometabolism and Nutrition

Hôpital Pitié-Salpêtrière

75013 Paris

karine.clement@psl.aphp.fr

Tel: 0033142177028

Fax: 0033142346993

\section{Shortened title}

Predicting diabetes remission

\section{Grants or fellowships}

Clinical Research Contracts/ Direction of Clinical Research Assistance Publique des Hôpitaux de Paris (PHRC 02076, CRC Macrophage Infiltration in Human Adipose Tissue, FIBROTA), the National program "Investissements d'avenir" with the reference ANR-10IAHU-05 (National Agency of Research), Fondation pour la Recherche Médicale (FRM), European Union Metacardis program (FP7). Nutriomics team received a grant from Institut Roche de Recherche en Médicine Translationnelle.

\section{Acknowledgements}


We thank Dr Florence Marchelli and Valentine Lemoine, APHP, Pitié-Salpêtrière, Paris, for the constitution of the database, Rohia Alili, UPMC, INSERM UMR_S U1166, Paris, for samples preparation and logistics, and Jean-Philippe Bastard and Soraya Fellahi, Functional Unit of Inflammatory and Metabolic Bio-markers, Tenon Hospital, Paris, for the measurement of C-peptide. We also thank Guillemette Ramey, Institut Roche de Recherche et Médecine Translationnelle, Boulogne-Billancourt, and Ludovic Le Chat, Institute of Cardiometabolism and Nutrition, Paris, for management of the scientific collaboration between ICAN and Roche. 


\begin{abstract}
Purpose

Statistical models and scores have been recently suggested to predict remission of type 2 diabetes after bypass surgery, but their relevance in routine clinical practice still needs evaluation. Our objective was to assess these methods on a French cohort and to compare them with other easy-to-use models.
\end{abstract}

\title{
Materials and Methods
}

We investigated a cohort of 84 diabetic obese subjects who underwent Roux-en-Y gastric bypass surgery. Diabetes remission one year after surgery was defined based on the American Diabetes Association criteria. We tested six methods from the literature and four other models to predict remission of diabetes after bypass surgery using pre-operative bioclinical parameters. Predictive methods for diabetes remission were assessed using cross-validation error rates when appropriate.

\section{Results}

$60 \%$ of the subjects had diabetes remission. Models from the literature had high error rates in our cohort (from 22.6 to $40.5 \%$ ), while published simple scoring systems behaved much better (15.9 and 16.7\%). Using other apprehensible models learned on our cohort did not improve the prediction error (from 17.2 to $19.9 \%$ ).

\section{Conclusions}

We showed that the scoring system DiaRem is easy to use and provide the best prediction error $(15.9 \%)$ compared to other methods. We additionally propose a DiaRem score threshold of $\leq 6$ for likely remission of a subject one year after surgery, which may be considered in clinical decision-making. 


\section{Key words}

type 2 diabetes, remission, bypass surgery, prediction, methods comparison 


\section{Introduction}

Gastric bypass surgery has been shown to improve type 2 diabetes (T2D) condition in severe obesity [1,2] and is increasingly used worldwide in the purpose of metabolic surgery [3]. Despite its recognized efficacy, operated patients respond differently to the intervention. T2D remission is observed in 41 to $100 \%$ of subjects after bypass surgery and the percentage of remission depends on the cohort under study as well as on the criteria used to define remission [4-6]. It is thus important for patients, clinicians and surgeons to identify methods that accurately predict patients' outcomes, and to test their relevance in clinical practice.

Attempts have been made to find pre-operative bioclinical parameters associated with T2D remission or to predict remission based on these parameters. Lower fasting plasma glucose and $\mathrm{HbA} 1 \mathrm{c}$ levels, shorter diabetes duration and persistent insulin secretion (as seen with higher C-peptide values and absence of insulin therapy), younger age, higher Body Mass Index (BMI) and lower prevalence of hypertension are common clinical variables that have been the most frequently found associated with T2D remission [7,8]. Models including logistic regression, decision trees or specific scoring systems have been used to predict T2D remission [9-13]. However, the predictive performances of these methods have not been validated in independent cohorts. They were often evaluated in only one cohort, using various definitions of T2D remission, and sometimes mixing different surgical procedures. In addition, most of the prediction errors were computed on the same dataset also used to build the model, which can lead to an overly optimistic error estimate.

Our purpose here was to assess these available models and scoring systems on a French cohort of morbidly obese diabetic subjects and compare them with other models built on our cohort using usual bioclinical parameters associated with $\mathrm{T} 2 \mathrm{D}$ remission. We focused on models that could easily be used by clinicians and that were based on established methods such as logistic regression and decision trees, but also considered penalization methods like 
lasso regression [14] or elastic nets [15]. We used a cross-validation estimate of the error rate to estimate more accurately the behavior of the predictor on a new cohort. 


\section{Materials and Methods}

\section{Anthropometric and biochemical parameters}

Eighty-four type 2 diabetic subjects with BMI $\geq 35 \mathrm{~kg} / \mathrm{m}^{2}$ were recruited in the Department of Nutrition, Center of Reference for Medical and Surgical Care of Obesity, at the Institute of Cardiometabolism and Nutrition (ICAN), Pitié-Salpêtrière Hospital (Paris, France). All subjects underwent gastric Roux-en-Y bypass (RYGB) surgery. T2D was defined as fasting glycemia $\geq 7 \mathrm{mmol} / \mathrm{L}$ or upon oral glucose tolerance test diagnostic (ie. glycemia at $120 \mathrm{~min}$

$>11 \mathrm{mmol} / \mathrm{L}$ ) or use of any anti-diabetic treatment. The diagnosis of type 1 diabetes was excluded by the clinical presentation of the diabetes and the absence of documented history of diabetic ketoacidosis. One year after RYGB, diabetes remission was defined in agreement with the American Diabetes Association (ADA) criteria of partial remission: no anti-diabetic drug, glycemia $<7 \mathrm{mmol} / \mathrm{L}$ and HbA1c $<6.5 \%$ [16]. Remission was assessed using the ADA partial criteria to take into account subjects with overall improvement of their glycemic status [6]. Blood samples were obtained after 12 hours of fasting prior to surgery and one year after. Glucose and HbAlc were measured according to standard methods used in routine care at Pitié-Salpêtrière hospital. Circulating C-peptide was measured using the Architect immunoassay (Abbot, USA). The ethic committee of the CPP Ile de France 1 approved the clinical investigations (NCT00476658, NCT01655017, NCT01454232).

\section{Statistical analysis}

Six methods from the literature were used: simple logistic and J48 decision tree models from Hayes et al. [9], two logistic regressions from Dixon et al. [10], and two scoring systems: one from Lee at al. and the DiaRem score from Still et al. [11,12] (see detail in Supplementary Information). In addition to those published methods, we applied logistic regression, decision trees, lasso regression [14] and elastic nets [15] on 10 pre-operative parameters 
usually associated with T2D remission: sex, age, BMI, fasting glycemia, HbAlc, hypertension, T2D duration, insulin therapy, number of anti-diabetic drugs and C-peptide value [7]. Decision trees and logistic regression hypothesize different kinds of data structures. Lasso regression, which is based here on logistic regression, allows automatic feature selection, but does not handle well correlated variables, while elastic nets are able to deal with them. Specific scoring systems are built from usual statistical models, but are much simpler to use, with the score being usually incremented when the value of a variable is below or above a predetermined threshold. More detail is given in Supplementary Information.

For logistic regression and decision trees a simple stratified $10 \times 10$ fold cross-validation was used to compute the prediction error. When model parameters needed to be estimated, i.e. thresholds for Lee and Still scoring systems and parameters for lasso regression and elastic nets, prediction error was computed using a nested stratified $10 \times 10$ fold cross-validation (Supplementary Figure 1). The internal cross-validation was used to estimate the parameters of the models and the external cross-validation was used to estimate the prediction error. All statistical analyses were done using the R software [17] and the "party" and "glmnet" packages $[18,19]$. 


\section{Results}

\section{Bioclinical parameters}

Among the 84 diabetic obese subjects who underwent RYGB surgery, $50(60 \%)$ achieved diabetes remission (DR subjects) one year after surgery whereas 34 (40\%) did not (DNR subjects). The patient's baseline bioclinical parameters are presented in Table 1. Gender and BMI were not different between groups. However, as expected, DNR subjects were older, had higher fasting plasma glucose, HbAlc and diabetes duration, as well as lower C-peptide values. DNR subjects also presented a higher prevalence of hypertension and took more antidiabetic drugs at baseline, especially insulin therapy.

\section{Scoring systems performed the best among state of the art methods}

We then compared state of the art methods for prediction of T2D remission one year after surgery on this cohort (Table 2). The Hayes and Dixon models featured good error rates in their original cohorts (between 12 and 16\%) but did not perform well on our French cohort with error rates between 22.6 and $40.5 \%$. The two scoring systems from Lee and Still did not provide prediction error in their publications, but we were able to extrapolate information from the published data (Table 2). Their methods behaved in a satisfactory manner on our cohort with close cross-validation error rates of 15.9 (DiaRem score, Still et al.) and 16.7\% (Lee et al.). We estimated the cross-validation optimized thresholds, that were 5 for Lee et al. (remission if score $\geq 5$ ) and 6 for the DiaRem score (remission if score $\leq 6$ ).

\section{Other readable methods do not improve the prediction error}

In an attempt to improve the cross-validated prediction error, we also built other easily interpretable models (logistic regression, decision trees, lasso regression and elastic nets) in our cohort using bioclinical parameters often associated with T2D remission (see Materials and Methods) (Table 2). Not surprisingly, we managed to improve the error on the training dataset with logistic regression to $7.1 \%$. However, this is not a proper estimate of the 
prediction error and, in the absence of an independent test dataset, we estimated the error by cross-validation. Here, the four methods displayed a similar behavior with elastic nets presenting the lowest error rate (17.2\%). None of the newly built models achieved better results than the simpler scoring systems. 


\section{Conclusion}

Different models have recently been suggested to predict T2D remission after gastric bypass surgery, but are not currently used in routine clinical practice to predict patient outcomes. These models appeared to provide relatively good prediction errors between 12 and $16 \%$ $[9,10]$, but only poorly predicted T2D remission one year after RYGB surgery in our cohort of 84 French diabetic obese subjects. This might be due to differences in the criteria used for T2D remission: Dixon et al. solely based the definition on HbA1c value after surgery, independently of glucose level or the use of anti-diabetic treatment [10]. Significantly higher error rates in additional cohorts can be expected when error rates were not estimated by cross-validation or on a separate test dataset. Hayes et al. indeed mentioned that they used cross-validation for their variable selection, but not for their error rate computation [9]. Specifically, in the case of insulin therapy, their J48 decision tree predicts non-remission of T2D for pre-operative fasting glycemia below $16.4 \mathrm{mmol} / \mathrm{L}$. Since elevated fasting glycemia is usually associated with non-remission [7], this prediction seems counter-intuitive and could reflect overfitting of the data.

Two scoring systems have also been recently proposed by Still et al. (DiaRem score) and Lee et al. to estimate the likelihood of T2D remission [11,12]. Although these scores were tested in several cohorts, no threshold for prediction or a prediction error estimate was provided in the original publications and we extrapolated information from the published data. The error for the DiaRem score was lower in the original publication [12] compared to Lee's (19.4\% vs 27.3\%) but it had been estimated from the training dataset, while Lee's score error was estimated from a test dataset, which makes them difficult to compare. In addition, Lee et al. excluded subjects with low C-peptide values from their protocol, which may have enriched the dataset for subjects who were more difficult to classify [11]. Interestingly, these scoring systems provided significantly better results on our cohort. Both scoring systems present low 
error rates (15.9 and $16.7 \%)$ when predicting remission one year after surgery according to the ADA partial criteria [16]. This means that out of 100 patients, we can obtain an accurate prediction for 84 of them. However, the DiaRem score from Still et al. requires easily accessible clinical information (age, HbAlc and anti-diabetic treatment), while the score from Lee et al. might be more difficult to obtain. This score requires age and BMI, but also diabetes duration which is frequently self-reported and difficult to assess, and C-peptide which is not always measured in routine care. Our paper brings additional information by providing a threshold for the DiaRem score, recommending to predict T2D remission of a subject one year after bypass surgery for a DiaRem score below or equal to 6 . We hope that this threshold may be useful in clinical practice, and it would be interesting to confirm its relevance in cohorts where the DiaRem score was already applied [12,20] and in others from different countries.

It should be noted that we did not manage to improve the prediction error using other classical methods that would be easy to use for clinicians. For example, current methods often fail in subsets of subjects who resolve their diabetes despite very high pre-operative HbA1c and the use of insulin therapy, most of them even achieving complete remission according to the ADA criteria (glycemia $<5.6 \mathrm{mmol} / \mathrm{L}, \mathrm{HbAlc}<6 \%$ ) [16]. Some other crucial parameters, such as compliance to therapy, could partly explain such situations but remain difficult to quantify properly.

We should point however to a few caveats of our approach. Firstly, the number of patients is relatively small. Secondly, we focused on T2D remission one year after bypass surgery, but it would be interesting to assess how these methods behave to predict longer term remission [5], as well as for different bariatric surgery techniques. In addition to that, only easily interpretable models were used, while more sophisticated methods might be needed to further improve the prediction accuracy. For example, support vector machines, which allow for 
overfitting control and non-linear separation, or random forests, which combine multiple decision trees in the hope of obtaining a better predictor, might be useful.

Within the limitations of the study, data suggest that a DiaRem Score below or equal to 6 would be a useful predictor of diabetes remission in subjects with T2D undergoing RYGB. 


\section{Conflict of Interest}

$\mathrm{AC}, \mathrm{CP}, \mathrm{GD}, \mathrm{JA}, \mathrm{JLB}, \mathrm{TS}$ and $\mathrm{KC}$ declare no conflict of interest. 


\section{Informed Consent}

Informed consent was obtained from all individual participants included in the study. 


\section{Human and Animal Rights}

All procedures performed in studies involving human participants were in accordance with the ethical standards of the institutional and/or national research committee and with the 1964 Helsinki declaration and its later amendments or comparable ethical standards. 


\section{References}

1. Ferchak CV, Meneghini LF. Obesity, bariatric surgery and type 2 diabetes--a systematic review. Diabetes Metab. Res. Rev. 2004;20:438-445.

2. Mingrone G, Panunzi S, De Gaetano A, Guidone C, laconelli A, Leccesi L, et al. Bariatric surgery versus conventional medical therapy for type 2 diabetes. N. Engl. J. Med.

2012;366:1577-1585.

3. Buchwald H. The Evolution of Metabolic/Bariatric Surgery. Obes Surg. 2014;1-10.

4. Pories WJ, Swanson MS, MacDonald KG, Long SB, Morris PG, Brown BM, et al. Who would have thought it? An operation proves to be the most effective therapy for adult-onset diabetes mellitus. Ann. Surg. 1995;222:339-350; discussion 350-352.

5. Davies SW, Efird JT, Guidry CA, Penn RI, Sawyer RG, Schirmer BD, et al. Long-term diabetic response to gastric bypass. J. Surg. Res. 2014;

6. Pournaras DJ, Aasheim ET, Søvik TT, Andrews R, Mahon D, Welbourn R, et al. Effect of the definition of type II diabetes remission in the evaluation of bariatric surgery for metabolic disorders. Br J Surg. 2012;99:100-103.

7. Adams ST, Salhab M, Hussain ZI, Miller GV, Leveson SH. Preoperatively determinable factors predictive of diabetes mellitus remission following Roux-en-Y gastric bypass: a review of the literature. Acta Diabetol. 2013;50:475-478.

8. Wang G-F, Yan Y-X, Xu N, Yin D, Hui Y, Zhang J-P, et al. Predictive Factors of Type 2 Diabetes Mellitus Remission Following Bariatric Surgery: a Meta-analysis. Obes Surg. 2014;

9. Hayes MT, Hunt LA, Foo J, Tychinskaya Y, Stubbs RS. A model for predicting the resolution of type 2 diabetes in severely obese subjects following Roux-en Y gastric bypass surgery. Obes Surg. 2011;21:910-916.

10. Dixon JB, Chuang L-M, Chong K, Chen S-C, Lambert GW, Straznicky NE, et al. Predicting the glycemic response to gastric bypass surgery in patients with type 2 diabetes. Diabetes Care. 2013;36:20-26.

11. Lee W-J, Hur KY, Lakadawala M, Kasama K, Wong SKH, Chen S-C, et al. Predicting success of metabolic surgery: age, body mass index, C-peptide, and duration score. Surg Obes Relat Dis. 2013;9:379-384.

12. Still CD, Wood GC, Benotti P, Petrick AT, Gabrielsen J, Strodel WE, et al. Preoperative prediction of type 2 diabetes remission after Roux-en-Y gastric bypass surgery: a retrospective cohort study. The Lancet Diabetes \& Endocrinology. 2014;2:38-45.

13. Ramos-Levi AM, Matia P, Cabrerizo L, Barabash A, Sanchez-Pernaute A, Calle-Pascual AL, et al. Statistical models to predict type 2 diabetes remission after bariatric surgery. J

Diabetes. 2014; 
14. Tibshirani R. Regression shrinkage and selection via the lasso. Journal of the Royal Statistical Society Series B. 1996;58:267-288.

15. Zou H, Hastie T. Regularization and Variable Selection via the Elastic Net. Journal of the Royal Statistical Society Series B. 2005;67:301-320.

16. Buse JB, Caprio S, Cefalu WT, Ceriello A, Prato SD, Inzucchi SE, et al. How Do We Define Cure of Diabetes? Dia Care. 2009;32:2133-2135.

17. R Core Team. R: A Language and Environment for Statistical Computing [Internet]. 2014. Disponible sur: http://www.R-project.org/

18. Hothorn T, Hornik K, Zeileis A. Unbiased recursive partitioning: A conditional inference framework. Journal of Computational and Graphical Statistics. 2006;15:651-74.

19. Friedman J, Hastie T, Tibshirani R. Regularization Paths for Generalized Linear Models via Coordinate Descent. J Stat Softw. 2010;33:1-22.

20. Aminian A, Brethauer SA, Kashyap SR, Kirwan JP, Schauer PR. DiaRem score: external validation. Lancet Diabetes Endocrinol. 2014;2:12-13.

21. Benjamini Y, Hochberg Y. Controlling the False Discovery Rate: A Practical and Powerful Approach to Multiple Testing. Journal of the Royal Statistical Society. Series B (Methodological). 1995;57:289-300. 


\section{Tables}

Table 1: Comparison between DNR and DR subjects

\begin{tabular}{l|cc}
\hline & DR $(\mathbf{n}=\mathbf{5 0})$ & DNR $(\mathbf{n}=\mathbf{3 4})$ \\
\hline Gender & $15 \mathrm{M}(30 \%)$ & $13 \mathrm{M}(38 \%)$ \\
Age (years) & $46.96 \pm 9.14$ & $54.47 \pm 11.02^{* *}$ \\
BMI (kg/m $\mathbf{2}^{\mathbf{2}}$ & $46.93 \pm 5.82$ & $46.1 \pm 6.62$ \\
Hypertension (N) & 29 yes $(58 \%)$ & 31 yes $(91 \%) * *$ \\
Glucose (mmol/L) & $7.25 \pm 1.8$ & $9.24 \pm 3.09 * *$ \\
HbA1c (\%) & $7.01 \pm 1.03$ & $8.21 \pm 1.32 * * *$ \\
C-peptide (ng/mL) & $4.04 \pm 1.64$ & $2.2 \pm 1.41 * * *$ \\
Diabetes duration (years) & $3.86 \pm 4.64$ & $14.21 \pm 7.63 * * *$ \\
Anti-diabetic treatments (N) & $1.28 \pm 0.99$ & $2.68 \pm 1.01 * * *$ \\
Insulin treatment (N) & 6 yes $(12 \%)$ & 22 yes $(65 \%)^{* * *}$ \\
\hline
\end{tabular}

Data are mean \pm sd. DR: subjects who resolved their diabetes one year after surgery. DNR: subjects who did not resolve their diabetes. N: number. M: males. Groups were compared with Student t-tests for quantitative parameters and Fisher exact tests for qualitative parameters. P-values were adjusted for multiple testing using the Benjamini-Hochberg procedure [21]. **p-value $<0.01, * * * p$-value $<0.001$. 
Table 2: Prediction errors of the different models in percentage

\begin{tabular}{l|ccc}
\hline & Train error & Test error & $\begin{array}{c}\text { Cross- } \\
\text { validation } \\
\text { error }\end{array}$ \\
\hline Hayes - SL & 13.4 & 31 & \\
Hayes - J48DT & $\mathbf{1 2 . 6}$ & 34.5 & \\
Dixon - LR6 & 16 & 40.5 & \\
Dixon - LR7 & $\mathbf{1 2}$ & $\mathbf{2 2 . 6}$ & \\
Lee - Score & $27.3^{\mathrm{a}}$ & $\mathbf{1 6 . 7}$ & $\mathbf{1 6 . 7}$ \\
Still - Score & $19.4^{\mathrm{b}}$ & $\mathbf{1 5 . 5}$ & $\mathbf{1 5 . 9}$ \\
LR & $\mathbf{7 . 1}$ & & 19.9 \\
DT & 13.1 & & 17.6 \\
Lasso & 14.3 & & 18.9 \\
EN & 13.1 & & $\mathbf{1 7 . 2}$ \\
\hline
\end{tabular}

Train error is the error from the original cohort. Test error is the error on our French cohort with no cross-validation. Cross-validation error is the error on our cohort using stratified $10 \times 10$ fold cross-validation. The 3 best results per column are in bold font. SL: simple logistic. J48DT: J48 decision tree. LR6: logistic regression for prediction of HbA1c $\leq 6$ one year after surgery. LR7: logistic regression for prediction of HbA1c $\leq 7$ one year after surgery. LR: logistic regression. DT: decision trees. EN: elastic nets. ${ }^{a}$ Deduced from the data provided in the paper using the best possible cut-off on the test dataset (remission is predicted if score $\geq 5$ ). ${ }^{b}$ Deduced from the data provided in supplementary material for the partial remission definition at 14 months using the best cut-off, but with the limitation that the score was already grouped by ranges (remission is predicted if score $\leq 7$ ). ${ }^{\mathrm{c}}$ All variables were included in the model. 